\title{
SAW Delay Line for Vibration Sensors
}

\author{
J. Filipiak ${ }^{a, *}$, L. Solarz $^{b}$ And G. SteCzko ${ }^{a}$ \\ ${ }^{a}$ Institute of Electronic and Control Systems, Technical University of Częstochowa \\ Armii Krajowej 17, 02-240 Częstochowa, Poland \\ ${ }^{b}$ Department of Civil Engineering, Military University of Technology \\ S. Kaliskiego 2, 00-908 Warsaw, Poland
}

\begin{abstract}
The paper presents an analysis of surface acoustic wave delay line. The line consists of two simple interdigital transducers placed on a ST-cut quartz plate. Mid-band operation frequency of the line is $74 \mathrm{MHz}$. The line will be applied for surface acoustic wave vibration sensor. At the immovable end of the plate there are electric signal feeds to both transducers. This is the cause of increase in signal value going directly between the transducers and the cause of line losses. By means of equivalent electric model of interdigital transducer a loss analysis of the line has been made at $50 \Omega$ load. The analysis allows to minimize line losses by matching the transducers to $50 \Omega$ impedance. This has been practically achieved by a design of transducer geometry and configuration matching $50 \Omega$ impedance, by means of inductance. An analysis of repeated operation characteristics of two interdigital transducers has been made. A signal going directly through capacitance between transducers and signals reflected from the edge of piezoelectric substrate have been presented. Results of theoretical analysis have been compared with experimental examinations.
\end{abstract}

PACS: 43.25.Fe, 77.65.Dq, 68.35.Iv

\section{Introduction}

Surface acoustic waves (SAW) in piezoelectric substrates are excited by interdigital transducers. The basic area of technical use of SAW are band-pass filters. They operate at a wide range of frequencies from several $\mathrm{MHz}$ to $2.5 \mathrm{GHz}$. They possess various frequency characteristics. As passive devices, they introduce losses of several dB. Loss value depends on filter design and piezoelectric substrate type. SAW filters are widely applied in telecommunications. SAW filters are also applied in SAW sensors measuring various physical quantities.

The sensors operation consists in measuring time delay changes alteration of surface wave propagation in SAW filter cased by a parameter that is being measured. For that reason, SAW filter plays the role of SAW delay line. Most researches are concerned with biochemical sensors and gas concentration sensors [1-4], as well as temperature, pressure and moment of force sensors [5-8]. Fewer focus on acceleration, vibration parameter, and stress sensors [9-13].

SAW delay lines are the basic element of SAW sensors. Depending on methods of measurement and type of measured parameter, various designs of delay lines are applied. Piezoelectric substrates of various physical properties are used to make the lines. Also, the lines are of various electric parameters. Most line designs are based on two cooperating interdigital transducers. In electrical terms, such a line is a passive four-terminal network. The distance between transducers is set by delay time of SAW delay line. SAW filter casing is encapsulated and connected to the circuit mass. Modeling line parameters

\footnotetext{
* corresponding author; e-mail: gem@gemsc.com.pl
}

and parasitic effects in line operation are common problems that need to be solved when designing SAW sensors.

Designs of SAW delay lines for vibration, acceleration and stress sensors differ from lines applied for sensors of other physical quantities. Figure 1a presents a sketch of a classic delay line and a SAW vibration sensor delay line. Figure $1 b$ presents vibration sensor delay line. SAW delay line is made on piezoelectric plate. One end of the plate remains immovable. The other free end of the plate can be loaded with seismic mass. The plate vibrates. Because of sensor plate movement, electrodes feeding electric signal to transducers (cumulative bars) should be placed on the immovable part of the plate. That will provide proper endurance of electric contacts to the electrodes. The electrodes are long. They are of particular effective resistance. Because of plate movement, its casing is larger than in classic SAW filters. This causes increase in signal going directly between input and output of SAW delay line (electromagnetic radiation coupling).

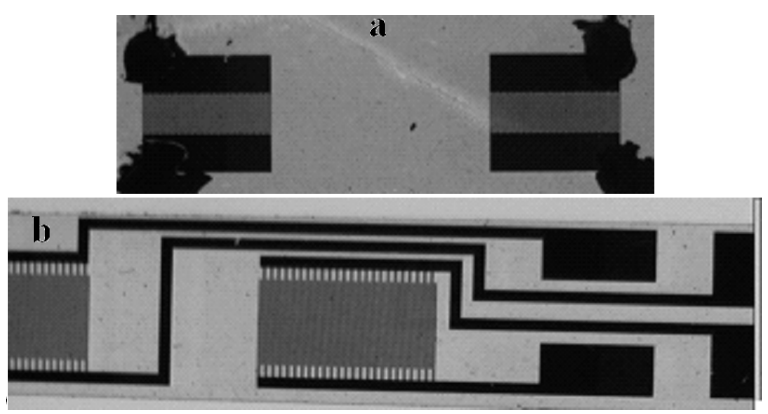

Fig. 1. SAW delay lines: classic (a), for vibration sensor (b). 
Quartz is often applied as piezoelectric substrate. ST-cut quartz has good temperature properties. Dependence of relative alteration in surface wave delay $\frac{\Delta \tau}{\tau}$ on temperature is described as follows:

$$
\frac{\Delta \tau}{\tau}=-34 \times 10^{-9}\left(T-T_{0}\right)^{2},
$$

where $T$ - temperature $(\mathrm{K}), T_{0}$ - return temperature (295 K for ST-cut quartz).

This property has been decisive in choosing ST-cut quartz as material for sensor plates. Quartz is a weak piezoelectric. Its electromechanical coupling coefficient value is low and amounts to $k^{2}=0.16 \%$. Relative dielectric permittivity is $\varepsilon=4.5$. The line has been designed as two cooperating identical simple periodic interdigital transducers with double electrodes. Such a structure of transducers allows to apply their operation on the third harmonic. Transducer electrode circuit is presented in Fig. 2.

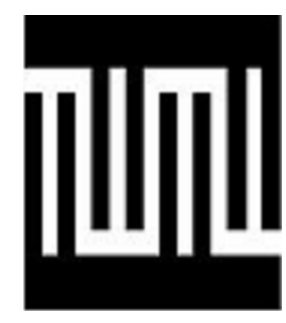

Fig. 2. Delay line transducer structure.

Low electromechanical coupling coefficient value for ST-cut quartz is the cause of considerable loss on mismatch of its input impedance to $50 \Omega$. In order to minimize the loss, interdigital transducer operation in conditions matching $50 \Omega$ impedance at frequency $74 \mathrm{MHz}$ has been chosen.

Line design will be discussed. We will analyze its basic parameters and parasitic effects in line operation. Results of experimental examinations of the line for vibration sensors will be presented.

\section{SAW delay line model}

Delay line composed of two cooperating interdigital transducers is a passive four-terminal network. Its electric parameters are line input and output admittances $Y_{N}, Y_{0}$ and transadmittance of two cooperating transducers $Y_{W}$. The line cooperates with electronic units as presented in Fig. 3. Parameters of electronic units are described by their impedances: $R_{\mathrm{g}}$ and $R_{0}$.

Equations describing cooperation of SAW delay line with electronic units are as follows:

$$
\begin{aligned}
& I_{N}=Y_{N} E_{N}+Y_{W} E_{O}, \\
& I_{O}=Y_{W} E_{N}+Y_{0} E_{O}, \\
& E_{N}=E_{\mathrm{g}}-I_{N} R_{\mathrm{g}}, \\
& E_{O}=-I_{O} R_{O} .
\end{aligned}
$$

From this system of equations we can determine frequency characteristic of the line [14]:

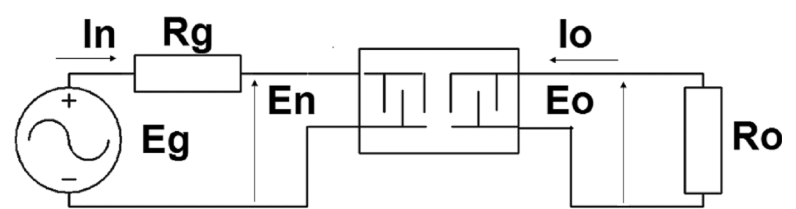

Fig. 3. SAW delay line cooperation with electronic units.

$$
H=\frac{E_{0}}{E_{\mathrm{g}}}=\frac{\frac{Y_{W} R_{0}}{\left(1+Y_{N} R_{\mathrm{g}}\right)\left(1+Y_{0} R_{0}\right)}}{1-\frac{Y_{W}^{2} R_{0} R_{\mathrm{g}}}{\left(1+Y_{N} R_{\mathrm{g}}\right)\left(1+Y_{0} R_{0}\right)}},
$$

which can be presented in the following way:

$$
\begin{aligned}
H & =\frac{Y_{W} R_{0}}{\left(1+Y_{N} R_{\mathrm{g}}\right)\left(1+Y_{0} R_{0}\right)} \\
& \times \sum_{n=0}^{\infty}\left[\frac{Y_{W}^{2} R_{0} R_{\mathrm{g}}}{\left(1+Y_{N} R_{\mathrm{g}}\right)\left(1+Y_{0} R_{0}\right)}\right]^{n} .
\end{aligned}
$$

The first term of an infinite series in form of

$$
H_{1}=\frac{Y_{W} R_{0}}{\left(1+Y_{N} R_{\mathrm{g}}\right)\left(1+Y_{0} R_{0}\right)}
$$

describes value of signal going between sending and receiving transducers in form of surface wave. It is an impulse frequency characteristic of two cooperating interdigital transducers. Whereas subsequent terms in an infinite series in expression (5) describe value of signals circulating between transducers and successively received by the receiving transducer. Because of the way they originated, we will call the signals multiple echoes. Their source is surface wave reflection from interdigital transducer. Surface wave reflection from receiving transducer coefficient will be described by the following dependence [14]:

$$
\Gamma=\frac{Y_{W} R_{0}}{1+Y_{0} R_{0}} .
$$

Thus value [14]:

$$
\frac{Y_{W}^{2} R_{0} R_{\mathrm{g}}}{\left(1+Y_{N} R_{\mathrm{g}}\right)\left(1+Y_{0} R_{0}\right)}=\frac{E_{0, n+1}}{E_{0, n}}
$$

describes ratio of signal echo amplitude $(n+1)$ to amplitude of $n$-times echo.

Signals circulating between transducers deform amplitude and phase characteristic of the line. That is why they are parasitic signals. Their level should be low so that their influence on filter characteristic would be negligible.

In order to calculate SAW delay line frequency characteristic presented in Fig. 3, it is necessary to calculate line input and output admittance $Y_{N}, Y_{0}$ and transadmittance of two cooperating transducers $Y_{W}$. Finding relations between the values and transducer geometry and piezoelectric substrate parameters is the fundamental issue connected with SAW filter designing technique. To solve the problem a Delta function model is used as well as equivalent circuits model and coupled mode model [15-17]. Out of the three basic methods of modeling only the substitute diagram model is in the shape of an elec- 
tric unit. Thus it is possible to directly apply it to analyze interdigital transducer cooperation with an electric matching unit. All mentioned electric parameters of interdigital transducer can be calculated by applying the substitute diagram model.

Let us present an analysis of delay line (Fig. 3) operation, made on the basis of equivalent circuits model [14]. One transducer has constant depth of electrode overlap. The second transducer (weighed) has variable electrode length. Transadmittance line is presented by the following dependence [14]:

$$
\begin{aligned}
Y_{W} & =\frac{\omega \varepsilon_{0} \varepsilon k^{2} \sin ^{2}\left(\frac{\alpha}{2}\right)}{2 P_{-\frac{\alpha}{\pi}}^{2}(-\cos \Delta)} \\
& \times\left\{\sum_{n=1}^{N} \mu(n) \exp \left(-\mathrm{j}\left(n-\frac{N+1}{2}\right) \alpha\right)\right\} \\
& \times\left\{\sum_{n=1}^{M} B_{n} \exp \left(-\mathrm{j}\left(n-\frac{M+1}{2}\right) \alpha\right)\right\} \\
& \times \exp \left(-\mathrm{j}\left(\gamma+\frac{M+N}{2}\right) \alpha\right),
\end{aligned}
$$

where

$$
\alpha=\frac{\pi \omega}{\omega_{1}}, \quad \Delta=\frac{\pi r}{p}, \quad \omega_{1}=\frac{\pi V}{p},
$$

$r$ - transducer electrode area in section length, $p$ section length, $V$ - surface wave velocity, $\gamma$ - wave phase shift in the area between transducers, $B_{n}$ - electrode overlap depth in weighed transducer, $\mu_{n}$ takes values 1, 0, 1 depending on straight transducer electrode polarization, $P_{-\frac{\alpha}{\pi}}(-\cos \Delta)-$ the Legendre polynomial.

Simple transducer coefficient of admittance is described by the following expression:

$$
\begin{aligned}
Y_{N} & =\mathrm{j} \omega C_{N}+\frac{\omega \varepsilon_{0} \varepsilon k^{2} L}{4 P_{-\frac{\alpha}{\pi}}^{2}(-\cos \Delta)} \\
& \times\left\{\mathrm{j} \sin \alpha \sum_{n=1}^{N} \mu^{2}(n)+2 \sin ^{2}\left(\frac{\alpha}{2}\right)\right. \\
& \left.\times\left[\sum_{l=1}^{N} \mu(l) \sum_{m=1}^{N} \mu(m) \exp (-\mathrm{j}|m-n| \alpha)\right]\right\} .
\end{aligned}
$$

Transducer admittance is a parallel connection of conductance $G_{P}$ of transducer static capacity $C_{N}$ and transducer dynamic capacity $C_{d}$ :

$$
Y_{N}=G_{P}(\omega)+\mathrm{j} \omega C_{N}+\mathrm{j} \omega C_{d} .
$$

For simple transducer of geometry presented in Fig. 2 conductance value is described by the following equation:

$$
G_{P}(f)=G_{0}\left(\frac{\sin (x)}{x}\right)^{2},
$$

where

$$
x=\frac{\pi N\left(\omega-\omega_{1}\right)}{\omega_{1}}, \quad G_{0}=\frac{4}{\pi} k^{2} C_{s} \omega_{1} N^{2},
$$

$C_{s}$ - transducer electrode pair capacity, $N$ - number of transducer electrode pairs.

Transducer capacity $C_{P}$ is sum of its static $C_{0}$ and dy- namic $C_{d}$ capacities. Dynamic capacity of simple transducer of geometry presented in Fig. 2 is described by the following dependence:

$$
\omega C_{d}=G_{0} \frac{\sin (2 x)-2 x}{2 x^{2}} .
$$

The above dependences allow to calculate all electric parameters of SAW delay line.

There are various reasons for loss caused by SAW delay line [15-17]. In the case of ST-cut quartz in given transducer design, the main reason for loss is transducer mismatch to impedance $50 \Omega$. To decrease the loss, interdigital transducer operation in conditions matching impedance $50 \Omega$ at frequency $74 \mathrm{MHz}$. On the basis of matching conditions analysis we can determine transducer electric parameters.

\section{SAW delay line matching unit}

The method of transducer matching is presented in Fig. 4. The element matching transducer of conductance $G_{P}$ and capacity $C_{P}$ to impedance $R_{\mathrm{g}}=50 \Omega$ is inductance $L_{1}$. Matching condition is emission of disposed power on transducer conductance $G_{P}$, taken from voltage source $E_{\mathrm{g}}$ of internal impedance $R_{\mathrm{g}}=50 \Omega$.

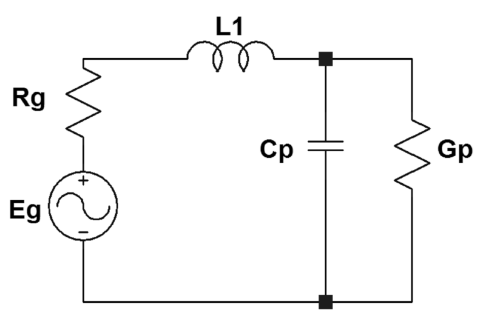

Fig. 4. Transducer matching circuit.

Power emitted on transducer conductance is changed into surface wave. It is described by the following dependence:

$$
P=\frac{E_{\mathrm{g}}^{2} G_{P}}{\left(1+R_{\mathrm{g}} G_{P}-\omega^{2} L_{1} C_{P}\right)^{2}+\left(G_{P} \omega L_{1}+R_{\mathrm{g}} \omega C_{P}\right)^{2}} .
$$

From the condition

$$
\frac{\mathrm{d} P}{\mathrm{~d} L_{1}}=0
$$

we can calculate maximum power emitted on transducer conductance. Its value is described by the dependence:

$$
P=\frac{E_{\mathrm{g}}^{2} G_{P}\left(G_{P}^{2}+\omega^{2} C_{P}^{2}\right)}{\left[R_{\mathrm{g}}\left(G_{P}^{2}+\omega^{2} C_{P}^{2}\right)+G_{P}\right]^{2}},
$$

which takes place when the following condition is fulfilled:

$$
L_{1}=\frac{C_{P}}{G_{P}^{2}+\omega^{2} C_{P}^{2}} .
$$

Disposed power, i.e. the highest power input from the source

$$
P_{\text {dys }}=\frac{E_{\mathrm{g}}^{2}}{4 R_{\mathrm{g}}} .
$$

From quantity 


$$
\frac{P}{P_{\mathrm{dys}}}=\frac{G_{P}\left(G_{P}^{2}+\omega^{2} C_{P}^{2}\right)}{4\left[\left(G_{P}^{2}+\omega^{2} C_{P}^{2}\right)+G_{P}\right]^{2}}=1
$$

we get a condition determining values of transducer electric parameters, conductance $G_{P}$ and capacity $C_{P}$ at which it is matched to impedance $50 \Omega$ at frequency $\omega$ :

$$
\frac{G_{P}}{G_{P}^{2}+\omega^{2} C_{P}^{2}}=R_{\mathrm{g}}=50 \Omega \text {. }
$$

We want to obtain transducer matching for frequency $\omega=\omega_{1}$. From expression (19), using dependences describing conductance $G_{P}$ and capacity $C_{P}(10),(11)$, we get quadratic equation regarding the amount of transducer electrodes $N$. After having solved the equation, we can calculate the amount of transducer electrode pairs from dependence

$$
N=\sqrt{\frac{\pi}{4(50 \Omega) k^{2} C_{\mathrm{K}} A \omega_{1}}-\left(\frac{\pi}{4 k^{2}}\right)^{2}},
$$

where $A$ - transducer aperture, $C_{\mathrm{K}}=0.05 \mathrm{pF} / \mathrm{mm}$ for quartz and assumed transducer design.

\section{Experimental results}

Using dependence (20), calculations of transducer geometry for ST-cut quartz have been made. Transducer aperture $A=2.5 \mathrm{~mm}$ has been assumed. For such parameters, a transducer composed of $N=25$ electrode pairs has been obtained. Transducer operation on the third harmonic at frequency $74 \mathrm{MHz}$ has been applied.

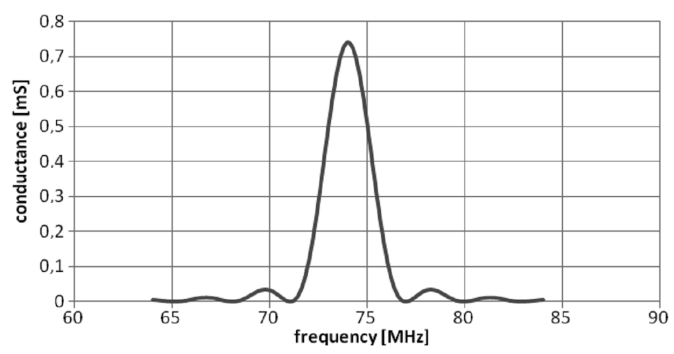

Fig. 5. Theoretical dependence of conductance of simple transducer composed of 25 double electrode pairs on ST-cut quartz.

Electrode width and gaps between electrodes amount to $16 \mu \mathrm{m}$, and acoustic surface wavelength is $37 \mu \mathrm{m}$. Figures 5 and 6 present results of theoretical calculations of transducer conductance and capacity in the function of frequency.

For frequency $74 \mathrm{MHz}$ transducer conductance is $0.74 \mathrm{mS}$ and capacity is $3.13 \mathrm{pF}$. For the quantities in dependence (16) inductance value has been calculated $\left(L_{1}=900 \mathrm{nH}\right)$ for which transducer matching condition to impedance $50 \Omega$ is fulfilled. Measuring coefficient of reflection from matched transducer allows to control matching interdigital transducer to impedance $50 \Omega$. Figure 7 presents alteration of reflection coefficient value from matched transducer in a unit of impedance $50 \Omega$ in frequency function. Inductance value of $L_{1}=900 \mathrm{nH}$ has been used for matching.

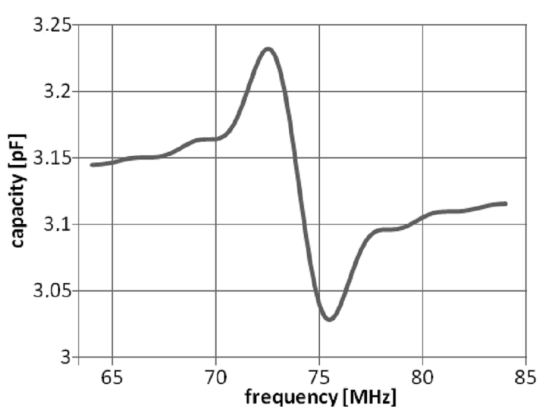

Fig. 6. Theoretical dependence of capacity of simple transducer composed of 25 double electrode pairs on ST-cut quartz.

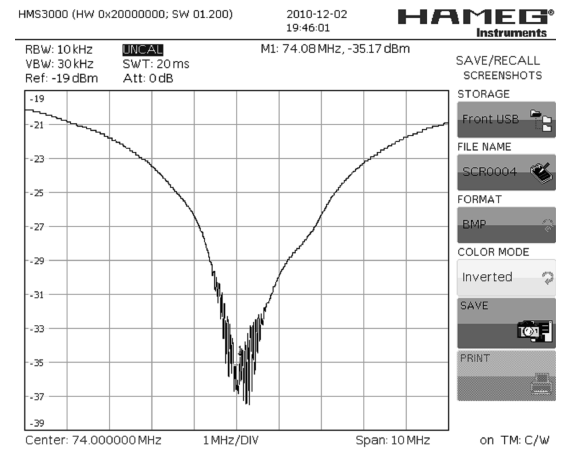

Fig. 7. Dependence of reflection coefficient from matched transducer in frequency function.

Quick-changing inequalities of reflection coefficient located around transducer operation frequency $(74 \mathrm{MHz})$ come from parasitic signals appearing in SAW delay line. Parasitic signals are: signal going directly between line input and output, multiple echoes, and signals reflected from crystal edge. These signals are received by receiving transducer at different times. To illustrate them, examinations have been made in unit presented in Fig. 8.

A signal in form of wave packet at frequency $74 \mathrm{MHz}$ has been sent onto line sending transducer. Packet length lower than line delay gives temporary separation of direct signal from usable signal. Figure 9 presents time run of signals in SAW delay line.

In Fig. 9, the following signals are presented, left to right: direct, usable, and other parasitic signals. Time run of signals in parasitic line after damping reflections from plate edge is presented in Fig. 10.

Liquidation of all signals with higher delay than usable signal attests to the lack of signals circulating be-

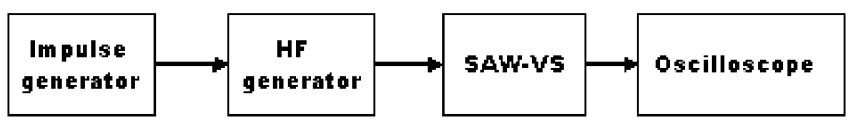

Fig. 8. Meter circuit for parasitic signals in SAW delay line. 


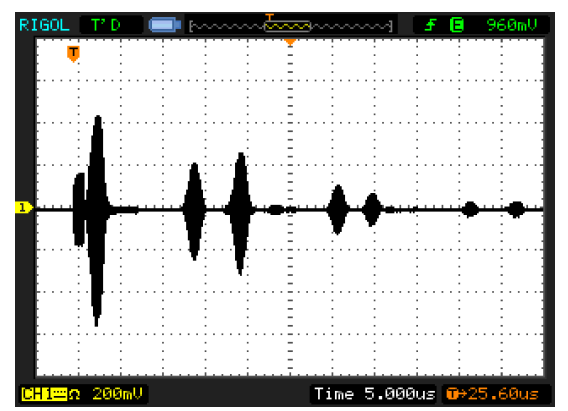

Fig. 9. Time run of signals in SAW delay line.

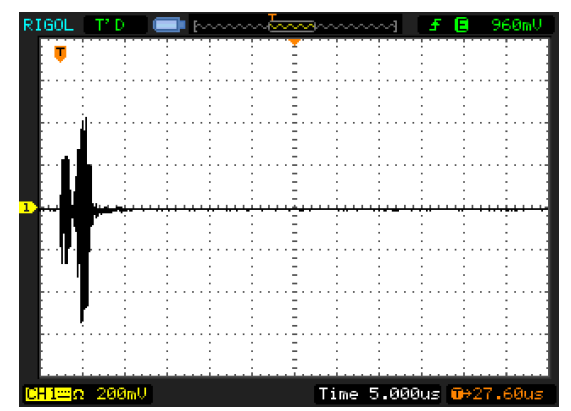

Fig. 10. Time run of signals in delay line after damping reflections from plate edge.

tween transducers. Figure 11 presents alteration of value of coefficient of reflection from transducer in a unit of impedance $50 \Omega$ in frequency function after signals reflected from plate edge have been liquidated.

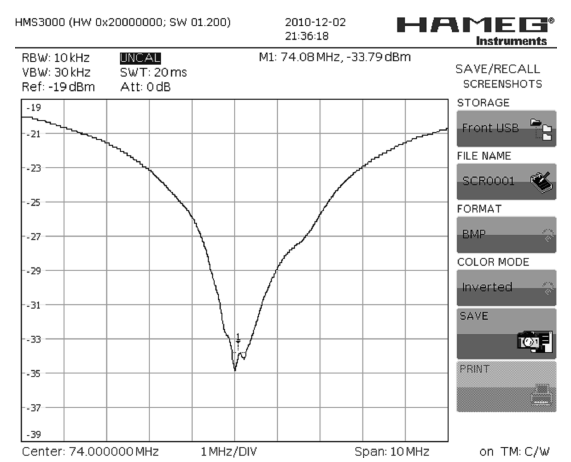

Fig. 11. Dependence of reflection coefficient from matched transducer in frequency function after signals reflected from plate edge have been liquidated.

Experimental amplitude frequency characteristics of SAW delay line is presented in Fig. 12.

Line losses after matching at frequency $74 \mathrm{MHz}$ amount to $24 \mathrm{~dB}$. SAW delay lines in vibration sensor.

\section{Conclusion}

We have presented a design and electric parameters of SAW delay lines on ST-cut quartz for vibration sensors.

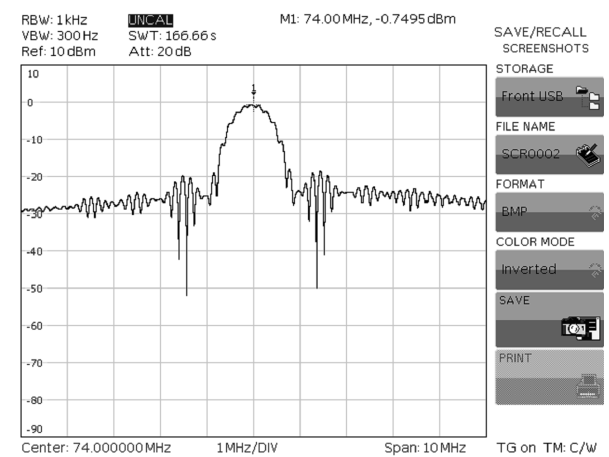

Fig. 12. Amplitude frequency characteristic of SAW delay line after transducer matching.

Simple periodic interdigital transducers operating on the third harmonic are applied in the lines. At operating frequency of $74 \mathrm{MHz}$, electrode width is $16 \mu \mathrm{m}$. Relatively wide electrodes facilitate line making. Line operation in matching conditions allow to decrease line loss to $24 \mathrm{~dB}$. The line operation model assumes ideal matching inductance and omits resistance of electrodes feeding the signal (cumulative bars) to the transducer. This has not influenced transducer matching to impedance $50 \Omega$ in any way. However, it can be the cause of higher line losses. Transducer matching unit taking into account coil magnification factor and resistance of cumulative bars is presented in Fig. 13. Resistance $R_{k}$ is a sum of inductance resistance, contacts and cumulative bars of the transducer. In the case of sending transducer, it is connected in series with internal resistance of the source modeling the cooperating electronic unit. In the case of receiving transducer, it is connected in series with load resistance. A signal feeding the interdigital transducer is decreased by voltage drop value on resistance $R_{k}$. The losses are described by value of relation $R_{k}$ to $50 \Omega$. When $R_{k}=0.5 \Omega$, the losses reach $1 \mathrm{~dB}$.

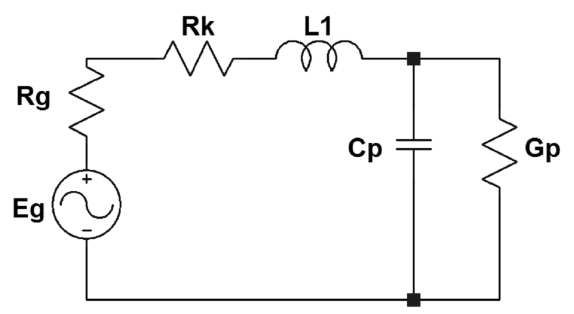

Fig. 13. Transducer matching circuit taking into account coil magnification factor and resistance of transducer cumulative bars.

Inductances used to match the transducer are of part of $\Omega$. Contacts made by ultrasonic welding are of similar value. Resistance of cumulative bar is estimated a few $\Omega$. That is why value $R_{k}$ is decisively higher for transducer with considerably long cumulative bars. Because of that, alteration of value of coefficient of reflection from transducer in a unit of impedance $50 \Omega$ in frequency function 
for transducer with long cumulative bars is lower than for transducer with short cumulative bars. It is caused by lowering quality factor of matching unit by higher resistance $R_{k}$. Losses caused by this resistance reach a few dB. Resistance $R_{k}$ lowers the level of multiple echo signal in SAW delay line. In delay line on quartz, the level of multiple echoes is practically negligible. On the basis of given results, it is possible to calculate multiple echo distribution in time. Making the Fourier transform (4) leads to dependence

$$
\begin{aligned}
h(t) & =\int_{-\infty}^{\infty}\left\{\frac{Y_{W} R_{0}}{\left(1+Y_{N} R_{\mathrm{g}}\right)\left(1+Y_{0} R_{0}\right)}\right. \\
& \left.\times \sum_{n=0}^{\infty}\left[\frac{Y_{W}^{2} R_{0} R_{\mathrm{g}}}{\left(1+Y_{N} R_{\mathrm{g}}\right)\left(1+Y_{0} R_{0}\right)}\right]^{n}\right\} \mathrm{e}^{\mathrm{j} 2 \pi f t} \mathrm{~d} f .
\end{aligned}
$$

For delay lines made on piezoelectric substrates the level of multiple echoes can be high. Figure 14 presents experimental run of multiple echoes of a line made on lithium niobate substrate.

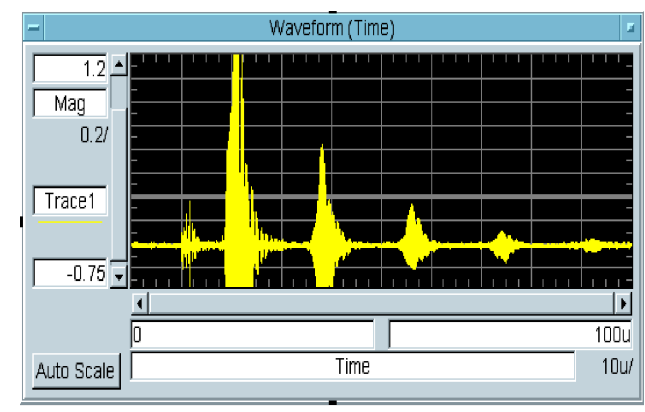

Fig. 14. Multiple echoes in a line made on niobate substrate.

Multiple echoes in SAW filters are parasitic signals. In the case of SAW delay line in a sensor the situation is different. If meter circuit operates with constant signal, then multiple echo signals add together with usable signal. Resultant signal is characterized by higher delay and amplitude modulation. Increase in signal delay leads to increase in sensor sensitivity. If meter circuit operates with impulse signal, then multiple echo signal can be applied as test signal. Such a solution increases sensor sensitivity.

However, the signal going directly between output and input of SAW delay line is parasitic signal. Decreasing its level is one of the fundamental problems connected with making SAW delay lines for vibration sensors. The pre- sented lines have been made for SAW vibration sensors applied in an electronic warning system [18].

\section{Acknowledgments}

This work was supported by the Polish Ministry of Science and Higher Education as a project "Vibration Warning System with SAW Vibration Sensors" included in the scientific budget of 2009-2011.

\section{References}

[1] H. Wohltjen, R. Dessy, Anal. Chem. 51, 1458 (1979).

[2] E. Gizeli, M. Liley, C.R. Love, H. Vogel, Anal. Chem. 69, 4808 (1997).

[3] C. Tyszkiewicz, T. Pustelny, Opt. Appl. 34, 507 (2004).

[4] P. Struk, T. Pustelny, K. Gut, K. Golaszewska, E. Kaminska, M. Ekielski, I. Pasternak, E. Łusakowska, A. Piotrowska, Acta Phys. Pol. A 116, 414 (2009).

[5] J. Filipiak, L. Solarz, G. Steczko, Acta Phys. Pol. A 120, 598 (2011).

[6] L.D. Clayton, E.F. EerNisse, IEEE Trans. Ultrason. Ferroelectr. Freq. Control 45, 1196 (1998).

[7] Q. Jiang, X.M. Yang, H.G. Zhou, J.S. Yang, Sensors Actuators A 118, 3 (2005).

[8] B. Drafts, Sensors 10, 1 (2000).

[9] E. Hauden, Arch. Acoust. 16, 47 (1991).

[10] J. Filipiak, L. Solarz, G. Steczko, Mol. Quant. Acoust. 28, 71 (2007).

[11] J. Filipiak, Surface Acoustic Wave Acceleration Sensors, Politechnika Częstochowska, Częstochowa 2006 , p. 121 (in Polish).

[12] J. Filipiak, C. Kopycki, Sensors Actuators 76, 318 (1999).

[13] J. Filipiak, L. Solarz, G. Steczko, Acta Phys. Pol. A 116, 302 (2009).

[14] J. Filipiak, L. Solarz, G. Steczko, Acta Phys. Pol. A 120, 593 (2011).

[15] L. Matthews, Surface Wave Filters, Wiley, New York 1997.

[16] P. Morgan, Surface Wave Devices for Signal Processing, Elsevier, London 1985.

[17] A. Oliner, Acoustic Surface Waves, Springer Verlag, Berlin 1978.

[18] J. Filipiak, L. Solarz, G. Steczko, Przeglad Elektrotechniczny 11a, 177 (2010) (in Polish). 